\title{
Torsion of a Rubber Cylinder
}

\author{
By Ronald S. Rivlin*
}

\begin{abstract}
It has been predicted theoretically that, in general, a right-circular cylinder of incompressible, highly elastic material, which is isotropic in its undeformed state, cannot be held in a state of pure torsional deformation by means of a torsional couple alone. In addition, normal surface tractions must be exerted over the plane ends of the cylinder. These normal surface tractions depend on the amount of torsion and on position on the plane ends of the cylinder. Experiments are reported here in which this phenomenon is observed in a rightcircular cylinder of pure gum compound. The dependence of the surface traction on amount of torsion and its distribution over the surface of the cylinder is studied by measuring the bulging of the rubber into small holes in a metal plate on one end of the cylinder.
\end{abstract}

\section{Introduction}

In a series of papers that is pending publication elsewhere, ${ }^{1}$ the mechanical properties of highly elastic materials, such as rubber, which can be assumed incompressible and isotropic in their undeformed state, are discussed in terms of a storedenergy function, $W$. This stored-energy function is specified in terms of the invariants of strain. It is shown that, once $W$ is specified in this manner, the forces, which must be applied in order to support any given state of deformation, can be calculated. This calculation has been carried out for the pure torsional deformation of a right-circular cylinder, for an assumed stored-energy function of simplest possible type and subsequently for any general form of stored-energy function.

The theory predicts that in order to produce a pure torsion in the cylinder, a torsional couple must be exerted on the plane ends of the cylinder, as in the classical theory of elasticity for small deformations. In addition, normal tractions must be exerted over these surfaces. The distribution of these normal tractions over the ends of the

*Guest worker at the National Bureau of Standards, representing the British Rubber Producers' Research Association, Welwyn Garden City Herts., England.

1 R. S. Rivlin, Phil. Trans. Roy. Soc. [A] (pending publication). cylinder and their dependence on the amount of torsion have been calculated for any specified form of the stored-energy function.

In this paper experiments are reported in which this distribution and dependence on amount of torsion is measured for a cylinder of pure-gum compound, on the assumption that the surface traction is measurable by the bulging of the rubber into small holes in a metal plate on one end of the cylinder. The results obtained are compared with those derived theoretically for two simple forms of the assumed stored-energy function. Agreement, within the accuracy of the experiments, is obtained if the stored-energy function has a form equivalent to that put forward for rubber by Mooney. ${ }^{2}$ This form involves two physical constants for the material.

\section{Specification of the Stored-Energy Function}

The stored-energy function for an ideal perfectly elastic material may be completely defined in the following manner. If a cube of the material, of unit edge, is subjected to a pure, homogenous deformation in which it is deformed into a cuboid

2 M. Mooney, J. Applied Phys. 11, 582 (1940). 
of dimensions $\lambda_{1}$ by $\lambda_{2}$ by $\lambda_{3}$, then the strain invariants $I_{1}, I_{2}$, and $I_{3}$ are defined as

$$
\left.\begin{array}{l}
I_{1}=\lambda_{1}^{2}+\lambda_{2}^{2}+\lambda_{3}^{2}, \\
I_{2}=\lambda_{2}^{2} \lambda_{3}^{2}+\lambda_{3}^{2} \lambda_{1}^{2}+\lambda_{1}^{2} \lambda_{2}^{2} \\
I_{3}=\lambda_{1}^{2} \lambda_{2}^{2} \lambda_{3}^{2} .
\end{array}\right\}
$$

If the material is isotropic in its undeformed state, the energy stored in the deformed cube is defined as the stored-energy function for the material. It must be a function of $I_{1}, I_{2}$, and $I_{3}$, provided it depends only on the state of deformation of the cube and not on the manner in which the deformation is reached. Once the form of this function is specified, the mechanical properties of any body of this material are, in principle, completely determinate and are purely a matter for calculation, provided such phenomena as internal friction and creep are negligible.

For an incompressible material, the volume of the cube considered is unaltered in the deformation, so that

$$
I_{3}=\lambda_{1}^{2} \lambda_{2}^{2} \lambda_{3}^{2}=1
$$

Then $W$, the stored-energy function, is given by

$$
W=W\left(I_{1}, I_{2}\right) .
$$

In general, the form of the function $W\left(I_{1}, I_{2}\right)$ will be such that when the body is undeformed $W=0$.

\section{Torsion of a Right-Circular Cylinder}

Let us consider a right-circular cylinder of radius, $a$, and length, $l$, of incompressible material, for which the stored-energy function, $W$, is given by eq 3. It has been shown that, in such a body, a pure torsion, in which each circular cross section of the cylinder is turned in its own plane through an angle $\phi(=\psi z)$ proportional to its distance $z$ from one end, can be maintained by the application of surface tractions alone. The necessary system of surface tractions is indeterminate to the extent of an arbitrary hydrostatic pressure, but if the surface tractions applied to the curved surface of the cylinder are zero, then the surface tractions that must be applied to the plane ends have been calculated as (1) tangential surface tractions, $\theta$, acting azimuthally in the deformed state of the cylinder (these effectively act as a torsional couple on the cylinder), and (2) normal surface tractions, $Z$.

$\theta$ and $Z$ are measured per unit area of the surfaces on which they act and their distribution over the plane ends of the cylinder and dependence on $\Psi$ are given by

and

$$
\Theta=2 \psi r\left(\frac{\partial W}{\partial I_{1}}+\frac{\partial \dot{W}}{\partial I_{2}}\right)
$$

$$
\begin{aligned}
& Z=-2 \psi^{2} a^{2}\left(\frac{\partial W}{\partial I_{2}}\right)_{r=a}+2 \psi^{2} \int_{a}^{r} r\left(\left(\frac{\partial W}{\partial I_{1}}-2 \frac{\partial W}{\partial I_{2}}\right)-\right. \\
& 2\left[\begin{array}{l}
\partial^{2} W \\
\partial I_{1}{ }^{2}
\end{array}+\left(3+\psi^{2} r^{2}\right)\left(\frac{\partial^{2} W}{\partial I_{1} \partial I_{2}}\right)+\left(2+\psi^{2} r^{2}\right) \frac{\partial^{2} W}{\partial I_{2}{ }^{2}}\right] d r+ \\
& 2\left[\frac{\partial W}{\partial I_{1}}-\left(\frac{\partial W}{\partial I_{1}}\right)_{r=a}\right]+4\left[\frac{\partial W}{\partial I_{2}}-\left(\frac{\partial W}{\partial I_{2}}\right)_{r=a}\right]
\end{aligned}
$$

in which $r$ is the distance of the point considered from the axis of the cylinder. $\partial W / \partial I_{1}, \partial W / \partial I^{2}$, $\partial^{2} W / \partial I_{1}^{2}$, etc. are, in general, functions of $I_{1}$ and $I_{2}$, which can be calculated if the form of $W\left(I_{1}, I_{2}\right)$ is known. For the deformation considered

$$
I_{1}=I_{2}=3+\psi^{2} r^{2}
$$

We shall consider here two particular choices of the form of the stored-energy function, $W$, for which eq 4 are considerably simplified.

In the series of papers (see footnote 1) mentioned above, particular attention was paid to the stored-energy function given by

$$
W=C_{1}\left(I_{1}-3\right),
$$

when $C_{1}$ is a physical constant characterizing the material. Materials for which eq 6 are valid were described as incompressible, neo-Hookean materials.

The particular interest residing in this choice of the stored-energy function rests on the facts that (1) it is the simplest choice that can be made, from the point of view of the formulation of a theory of large elastic deformations; (2) it is the form derived by Treloar, ${ }^{3}$ for an ideal rubber, on the basis of the kinetic theory of large elastic deformations.

Equation 6 is a particular form, obtained by making $C_{2}=0$, of the relation

$$
W=C_{1}\left(I_{1}-3\right)+C_{2}\left(I_{2}-3\right),
$$

${ }_{3}^{3}$ L. R. G. Treloar, Trans. Faraday Soc. 39, 241 (1943). 
which was first suggested by Mooney (see footnote 2 ) as suitable for describing the elastic properties of rubber. It is noted that eq 7 involves two physical constants for the material, $C_{1}$ and $C_{2}$.

If eq 7 is introducted into eq 4 , we obtain

and

$$
\Theta=2 \psi r\left(C_{1}+C_{2}\right)
$$

$$
Z=-2 \psi^{2} a^{2} C_{2}-\psi^{2}\left(a^{2}-r^{2}\right)\left(C_{1}-2 C_{2}\right) .
$$

The equations for $\theta$ and $Z$, when $W$ takes the form of eq 6 , can be obtained by putting $C_{2}=0$ in eq 8.

We see that if the physical properties of the material are represented by either eq 6 or 7 , the force, $Z$, that must be exerted at each point is proportional to the square of the amount of torsion. If eq 6 applies, $Z$ must everywhere have the nature of a thrust (as $C_{1}$ is essentially positive), which is distributed over the diameter of a plane and as shown in figure 1 (a). If eq 7 applies, then $Z$ does not vanish at the periphery (where $r=a$ ), and provided $C_{2}$ is positive and sufficiently small compared with $C_{1}$, its distribution will have the form shown in figure 1 (b).

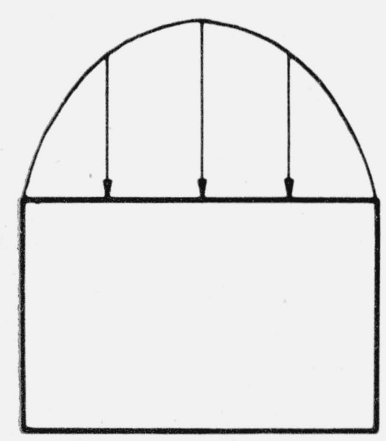

a

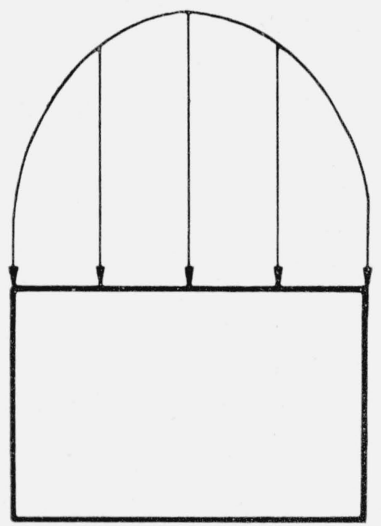

b
FIGURE 1.-Distribution of normal surface traction over plane ends of cylinder in pure torsion.

(a) when $W=C_{1}\left(I_{1}-3\right)$, and (b) when $W=C_{1}\left(I_{1}-3\right)+C_{2}\left(I_{2}-3\right)$.

\section{Experimental Procedure}

The experiments which will be described in this paper have as their object the study of the distribution of the normal force, $Z$, defined in the previous section, and its dependence on the amount of torsion.

In a preliminary qualitative experiment a rubber cylinder of pure gum compound, with brass plates bonded onto its plane ends, was subjected to a torsional couple by means of a lever attached to one of the ends, while the other end was held in a vise. It was found that unless a longitudinal thrust was intentionally exerted parallel to the axis of the cylinder, the cylinder elongated, its axial cross section before and after torsion being shown roughly in figure 2 . This simple experiment
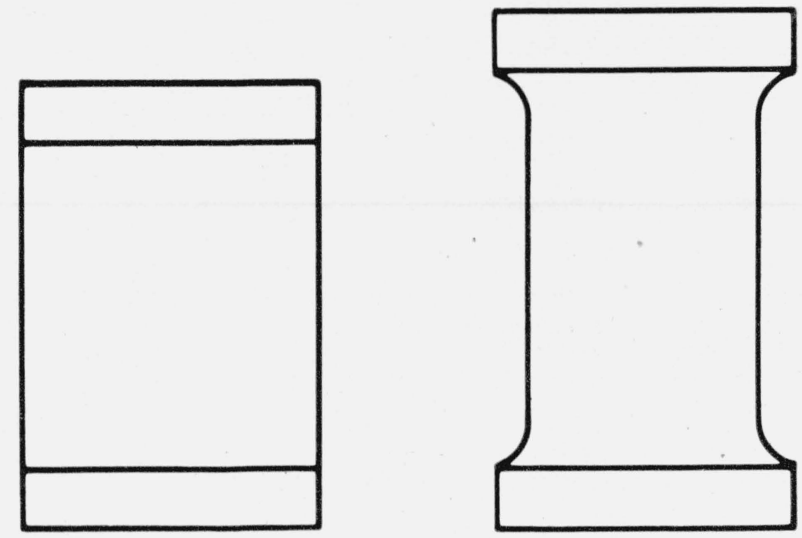

Figure 2.-Axial cross section of cylinder before and after twisting by couple.

bears out the mathematical conclusion that a pure torsional couple does not give rise to a pure torsion. Clearly, if a pure torsion is to be obtained, normal compressive forces, whose resultant must have the nature of a thrust, must be exerted over the plane ends of the cylinder. This is in general agreement with the theoretical predictions outlined in the previous section.

Consequently, an apparatus was designed in which the pure-gum cylinder, to whose plane ends brass disks are bonded, is restrained from elongating. So, when one disk is rotated with respect to the other by the application of a torsional couple, the longitudinal thrusts required for the production of a pure torsion in the rubber cylinder are automatically called into play. The upper brass disk, $A$, contains five circular holes, each $1 / 2-$ in. in diameter, varying in distance from the center and spaced as shown in figure 3 , in order to have as little mutual interaction as possible. The lower disk, $B$, has rigidly attached to it a steel disk, $C$.

The assembly of rubber cylinder and disks $A$, $B$, and $C$ is held in a robust steel frame, $D$, as shown in figure 4 . The upper disk is rigidly attached to the frame and the lower disk is capable of being rotated in its own plane about its axis, by means of a crowbar which is introduced into one of 
a series of holes in steel disk $C$. Provision is made for holding the cylinder in a state of torsion by means of steel pegs, which can be introduced into one of the holes, $E$, in the frame. A scale is also provided for measuring the angle through which the lower disk is rotated.

When this disk is rotated, the rubber bulges slightly in the holes in disk $A$, and the amount of this bulging is assumed to be proportional to the thrust which would have to be exerted to prevent the bulging.

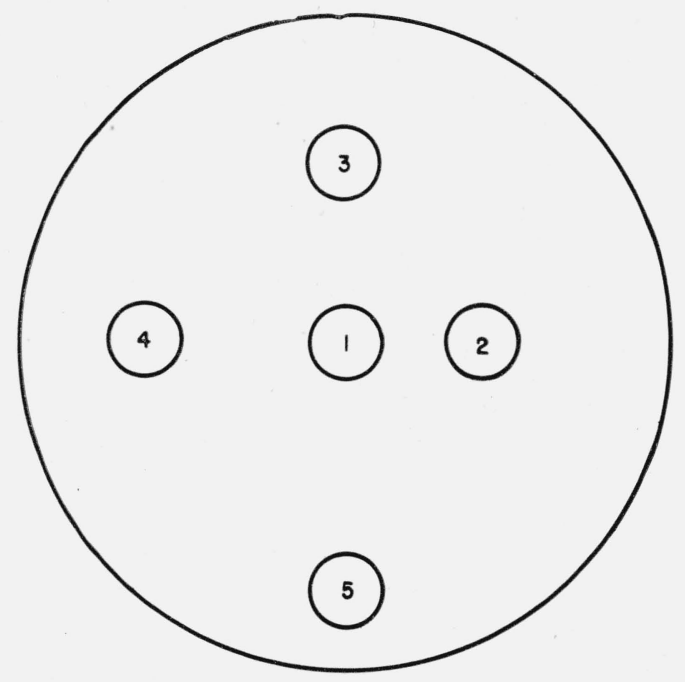

Figure 3.-Upper brass disk.

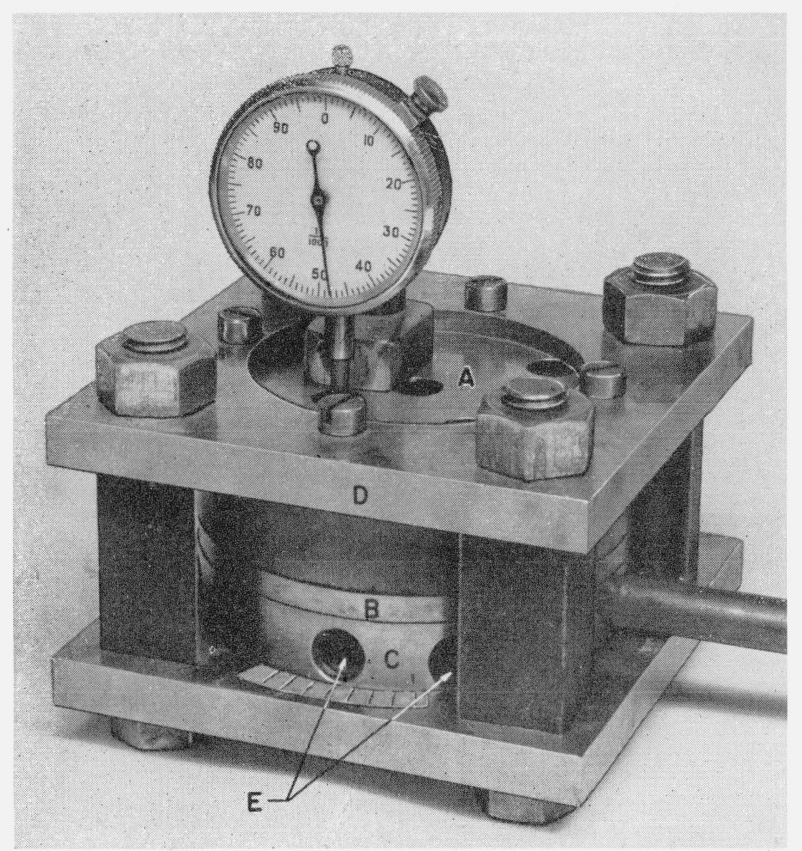

Figure 4.-The torsion apparatus.
The bulging represents a small deformation superposed on a relatively large torsional deformation. At each of the holes this torsional deformation approximates to a state of simple shear of an amount depending on the amount of the torsion and the position of the hole considered. The assumption that the amount of bulging is proportional to the normal force tending to produce it, is equivalent to an assumption that the former does not depend greatly on the state of simple shear on which the bulging deformation is superposed. There are certain theoretical reasons for assuming that this is approximately valid in the experiments described here.

In order to produce a simple extension in a cylinder superposed on the simple torsion, an additional normal surface traction must be exerted over each of the plane ends. If the stored-energy function of the material for the cylinder has the form of eq 6 , it has been shown (see footnote 1 ) that this additional normal surface traction is independent of the amount of torsion and of position on the surface of the cylinder. The stress distribution in the neighborhood of the hole is complex, but for small deformations it is reasonable to assume that the amount of bulge in each hole is dependent only on the force tending to produce the bulging and not on the amount of torsion or position of the hole, except insofar as this force is determined by them. This conclusion should be approximately valid if the stored-energy function has the form of eq 7 , provided $C_{2}$ is small compared with $C_{1}$. However, there is no reason to believe that it should be true for every possible form of the stored energy function.

The amount $d$ of the bulging in each of the holes is measured at the center of the hole, for various angles of rotation, $\theta$, of disk $B$, by means of a dial gage. The top of disk $A$ is ground flat and user as a reference plane in carrying out this measurement.

\section{Experimental Results}

If eq 8 is valid for $Z$, then the relation between $d$ and $\theta$ should be

$$
d=K \theta^{2}\left[2 a^{2} C_{2}+\left(a^{2}-r^{2}\right)\left(C_{1}-2 C_{2}\right)\right],
$$

where $K$ is some constant of proportionality, $r$ is now taken as the distance of the center of the hole from the center of the disk. This assumption is justified if the radius of each hole is small compared 
with that of the cylinder, but it is impracticable to make it too small as the values of $d$ are thereby reduced. The repeatability with which $d$ could be measured in the experiment was estimated as $\pm 0.002 \mathrm{in}$. using the same dial gage. The accuracy with which $\theta$ could be measured in the arrangement employed was $\pm 1 / 2^{\circ}$.

The gum compound used had the following composition in parts by weight:

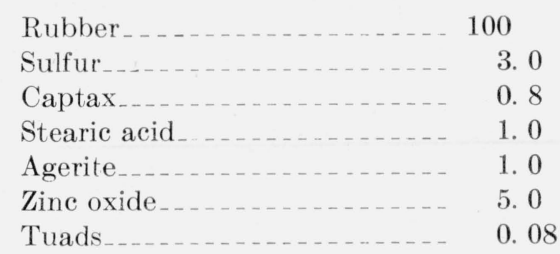

The mix was vulcanized for $30 \mathrm{~min}$ at $134^{\circ} \mathrm{C}$.

In order to produce a strong bond between the brass plates, a "tie gum" layer, less than $1 / 32$ in. thick, was introduced between the main rubber cylinder and each of the brass plates and a cement marketed as "Reanite, No. 3615" was employed. The composition of the "tie gum" layer was, in parts by weight,

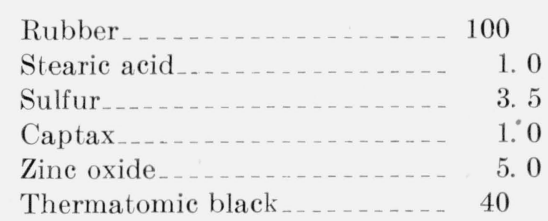

The rubber cylinder employed had a radius of 2.12 in. and a length of 1 in. The holes in disk $B$ each had a diameter of $1 / 2$ in., and the distances, $r$, of their centers from the center of the disk are:

\begin{tabular}{|c|c|c|c|}
\hline $\begin{array}{l}\text { Hole num- } \\
\text { ber }\end{array}$ & 2 & 3 & \\
\hline$r$, in in. . 0 & 0.85 & 1. 18 & 1. 47 \\
\hline
\end{tabular}

Equation 9 suggests that the results of the experiments be plotted as a relation between $d$ and $\theta^{2}$ on linear graph paper. This should yield a straight line passing through the origin for each hole.

This is done in figure 5 for each of the five holes, and it is seen that the linear relations between $d$ and $\theta^{2}$ of eq 9 are borne out within the accuracy of the experiment.

The slope, $m$, of the straight line obtained for each hole is the value of $K\left[2 a^{2} C_{2}+\left(a^{2}-r^{2}\right)\right.$ $\left.\left(C_{1}-2 C_{2}\right)\right]$ for that hole, and if this is plotted
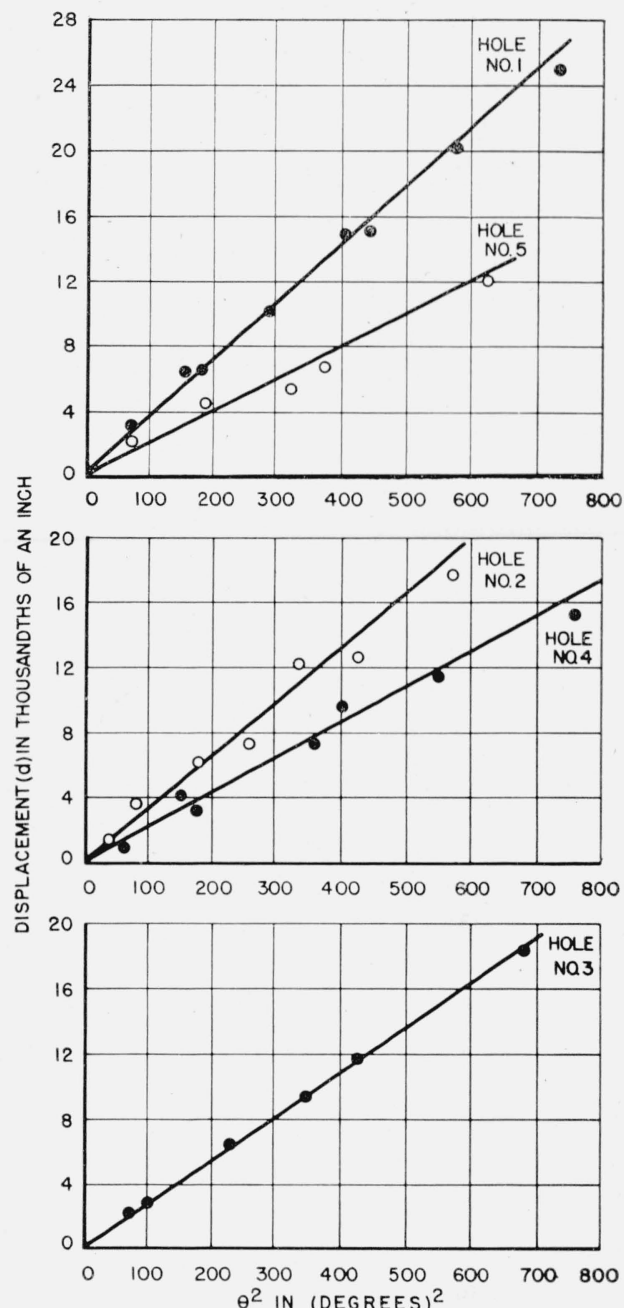

Figure 5.--Relation between displacement and the square of the angle of torsion.

against $\left(a^{2}-r^{2}\right)$, a straight line should be obtained, which passes through the origin if $C_{2}=0$ and otherwise gives an intercept of $2 a^{2} C_{2} K$ on the $m$-axis. If $m$ is plotted against $\left(a^{2}-r^{2}\right)$ as in figure 6 , it is seen that, within the accuracy of the experiment, the relation eq 9 between $d / \theta^{2}$ and $\left(a^{2}-r^{2}\right)$ is again borne out.

If $m_{0}$ is the intercept of the straight line in figure 6 on the $m$-axis and $b$ is its slope, we have $m_{0}=2 a^{2} C_{2} K$ and $b=K\left(C_{1}-2 C_{2}\right)$, whence

$$
\frac{C_{1}}{C_{2}}=\frac{2 b}{m_{0}} a^{2}+2
$$

From measurements on figure 6, we obtain $m_{0}=10 \times 10^{-6}$ in. deg. $^{-2}$, and $b=5.65 \times 10^{-6}$ in. $^{-1}$ deg. $^{-2}$, so that $C_{1} / C_{2}=7.1$. 


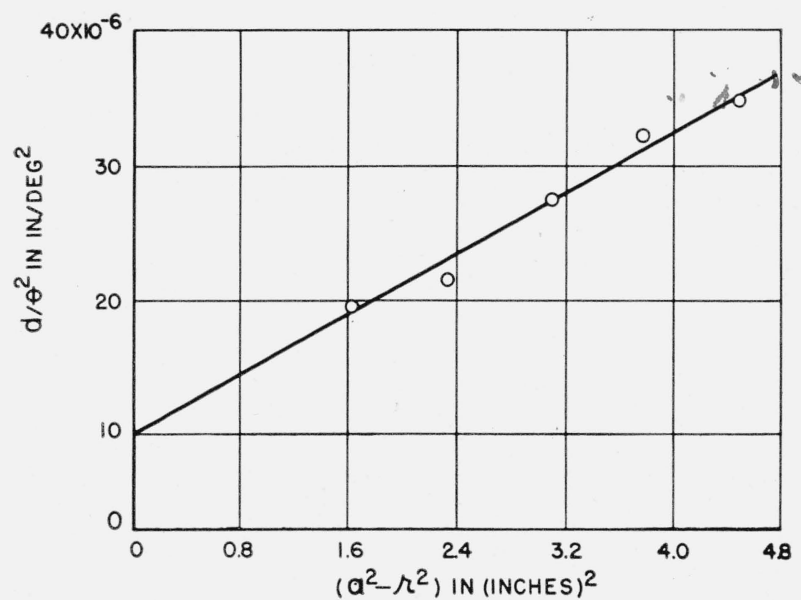

Figure 6.-Relation between slopes of lines in figure 5 and positions of holes.

The equation showing the relation $d / \theta^{2}=K\left[2 a^{2} C_{2}+\left(a^{2}-r^{2}\right)\left(C_{1}-2 C_{2}\right)\right]$

Mooney (see footnote 2) uses the constants $C_{1}$ and $C_{2}$ to define three additional constants as follows: $G=2\left(C_{1}+C_{2}\right) ; \quad \mathrm{H}=2\left(C_{1}-C_{2}\right) ;$ and $\alpha=\mathrm{H} / \mathrm{G}$. He then shows that constant $G$ is the modulus of rigidity of the material. Constant $\alpha$ is named the coefficient of asymmetry. From the definitions it follows that $\alpha=\left(C_{1} / C_{2}-1\right) /$ $\left(C_{1} / C_{2}+1\right)$.

Consequently, the experimental results presented here lead to a value of 0.75 for the coefficient of asymmetry of the pure-gum compound studied. This is somewhat larger than the values found for two compounds studied by Mooney. Further work would be required to establish the variation of $\alpha$ with the nature of the compound.

\section{Conclusions}

The experiments bear out the general conclusion of the theory that in order to produce pure torsion in a rubber cylinder, normal surface tractions must be exerted on the plane ends and that these surface tractions vary with the amount of torsion and the position on the plane end of the cylinder. If the assumption is made that the amount of bulging in each hole is proportional to the force 5 producing it and does not depend on the state of local strain on which the bulging is superposed, then the results of the experiments are in accordance with the assumption of an expression of the form of eq 7 for the stored-energy function of the rubber. It has already been pointed out by Mooney that a stored-energy function of this form describes the linear shearing stress vs. amount of shear relation for rubber in simple shear, with a fair measure of accuracy, up to reasonably large deformations. Also, Treloar ${ }^{4}$ has recently obtained results in agreement with this for pure, homogeneous strain of a sheet of rubber.

The form of eq 6 for the stored-energy function is that derived from the kinetic theory of rubberlike elasticity. Load versus deformation relations derived from this have been compared with experimental results for simple types of deformation and have yielded fairly good agreement, depending on the type of deformation employed.

It appears then that eq 6 for the stored-energy function provides a first approximation to the stored-energy function for vulcanized rubber and that a second approximation is provided by eq 7 . The differences between these forms are more or less accentuated depending on the type of deformation.studied.

This work forms part of a program of fundamental research on rubber undertaken by the Board of the British Rubber Producers' Research Association. The work was carried out at the National Bureau of Standards, Washington, D. C., where the author is at present a guest worker. The author is indebted to W. L. Holt of the National Bureau of Standards for advice and assistance in connection with the mechanical design of the apparatus.

\section{Washington, February 25, 1947.}

\footnotetext{
${ }_{4}^{4}$ L. R. G. Treloar, Trans. Faraday Soc. (pending publication).
} 